\title{
Impact of COVID-19: perspectives from gastroenterology
}

\author{
Shu Wen $\underline{T a y}^{1, *}$, MRCP, Kevin Kim Jun $\underline{T e h}^{1, *}$, MRCP, Lai Mun $\underline{\text { Wang }}^{2,3}$, FRCPEd, FRCP, Tiing Leong $\underline{\text { Ang }}{ }^{1,4,5}$, fRCPEd, FAMS
}

\section{OVERVIEW}

A novel human coronavirus, severe acute respiratory syndrome coronavirus 2 (SARS-CoV-2), that resulted in an outbreak of respiratory disease was first reported in Wuhan, China, in December 2019. (1) Coronavirus disease 2019 (COVID-19) was declared a pandemic by the World Health Organization on 11 March 2020. By the end of March 2020, almost a million people had been afflicted. ${ }^{(2)}$ This reflects the ease of transmission amid global interconnectivity. Most infected patients have mild disease. When severe disease with viral pneumonia occurs, it may rapidly progress to acute respiratory distress syndrome, resulting in respiratory failure and death. Current management strategies focus on public health and infection control measures to contain the disease, and respiratory support and treatment of severe cases. SARS-CoV-2 infection is of relevance to the field of gastroenterology due to gastrointestinal (GI) involvement and manifestations, ${ }^{(3)}$ its impact on vulnerable patients, ${ }^{(4)}$ and the risk of transmission during $\mathrm{Gl}$ endoscopy procedures. ${ }^{(5)}$

\section{GASTROINTESTINAL MANIFESTATIONS}

Human coronaviruses are known to cause both respiratory and Gl symptoms. ${ }^{(6)}$ Entry of coronaviruses into host cells is mediated by surface spike glycoproteins, which bind to angiotensin-converting enzyme 2 (ACE-2) receptors on human host cells. ${ }^{(7)}$ Immunohistochemistry studies have revealed that ACE-2 receptors are abundantly present in lung alveolar Type 2 cells and the enterocytes of the small intestine, with staining confined to the brush border in the latter. ${ }^{(8)}$ Infection of ACE2 receptors that express absorptive enterocytes can result in diarrhoea. SARS-CoV-2 RNA has been detected in stool samples, raising the possibility of faecal-oral transmission. ${ }^{(9)}$ Hence, although direct droplet transmission is probably the most important route of transmission, faecal excretion with environmental and fomite contamination may also contribute to viral transmission. This highlights the importance of good sanitation and proper hand hygiene.

The majority of patients who are diagnosed with COVID-19 present with a combination of fever and respiratory symptoms. However, Gl symptoms do occur in some and may cause diagnostic confusion. The actual prevalence rates of reported Gl symptoms differ across published studies, and this may reflect true differences in the studied populations, the phase of the disease being captured or study design limitations, as these are all retrospective observational studies. ${ }^{(10-13)}$ Diagnosis of Gl symptoms arising from COVID-19 infection is obvious in patients with epidemiological risk factors who present first with respiratory symptoms, with or without fever, within 14 days of exposure to the disease. A multicentre study of 1,099 patients from China reported a median incubation period of 4 (interquartile range 2-7) days. ${ }^{(13)}$ With the onset of local transmission, where there is an absence of exposure to known clusters, Gl symptoms may pose a diagnostic challenge. Fortunately, current epidemiological data suggests that fever and respiratory symptoms generally predate Gl symptoms and are the dominant symptoms. Thus, we should look out for these features during systemic review.

Table I summarises the occurrence of Gl symptoms in published studies by contextualising them in terms of the predominant symptoms of fever and respiratory symptoms. ${ }^{(10-13)}$ Recognising these Gl symptoms as being part of the acute infection can help to decrease the number of unnecessary Gl investigations during active disease. Studies by Chen et al, ${ }^{(10)}$ Wang et $\mathrm{al}^{(11)}$ and Guan et $\mathrm{al}^{(12)}$ reported the occurrence of Gl symptoms at admission to hospital, but did not explore the temporal relationship between the onset of $\mathrm{Gl}$ symptoms and fever/respiratory symptoms. A study by Fang et al specifically examined GI manifestations that developed 1-10 days after disease onset. Reported Gl symptoms included nausea (29.4\%, $59 / 201)$, vomiting $(15.9 \%, 32 / 201)$, abdominal pain $(6.0 \%$, $12 / 201)$ and diarrhoea $(49.5 \%, 146 / 295)$. After the exclusion of possible drug-related diarrhoea, the incidence of diarrhoea remained high at $22.2 \% .{ }^{(13)}$

Liver function test (LFT) abnormalities have also been reported. These abnormalities may reflect systemic inflammation or adverse effects of medications, or may be the consequence of organ failure. The virus itself does not result in acute hepatitis. Fang et al reported elevations in serum alanine aminotransferase $(\mathrm{ALT})$, aspartate aminotransferase (AST) or bilirubin levels in $39.1 \%(119 / 304)$ of patients at admission. However, those with ALT or AST $\geq 80 \mathrm{U} / \mathrm{L}$ accounted for only $7.9 \%$ (24/304) and $6.3 \%(19 / 304)$ of the patients, respectively. Only $2.0 \%$ (6/304) of patients had elevated serum bilirubin level. The prevalence of LFT abnormalities was significantly higher in the critically ill group compared to the non-critically ill group. ${ }^{(13)}$ Another study suggested that severe LFT abnormalities are due to adverse

${ }^{1}$ Department of Gastroenterology and Hepatology, ${ }^{2}$ Pathology Section, Department of Laboratory Medicine, Changi General Hospital, ${ }^{3} \mathrm{Pathology}$ ACP, ${ }^{4} \mathrm{Medicine}$ ACP, SingHealth Duke-NUS Academic Medical Centre, ${ }^{5}$ Yong Loo Lin School of Medicine, National University of Singapore, Singapore

*These authors contributed equally as first authors.

Correspondence: Prof Tiing Leong Ang, Chief and Senior Consultant, Department of Gastroenterology and Hepatology, Changi General Hospital, 2 Simei Street 3, Singapore 529889. ang.tiing.leong@singhealth.com.sg 
Table I. Gastrointestinal symptoms as compared to fever and respiratory symptoms in COVID-19 patients in the published literature.

\begin{tabular}{|lllll|}
\hline Clinical symptom & Chen et $\mathbf{a l}^{(\mathbf{1 0})}(\mathbf{n}=\mathbf{9 9})$ & Wang et $\mathbf{a l}^{(\mathbf{1 1 )})}(\mathbf{n}=\mathbf{1 3 8})$ & $\mathbf{G u a n ~ e t ~ a l}^{(\mathbf{1 2})}(\mathbf{n}=\mathbf{1 , 0 9 9 )}$ & Fang et al $^{(\mathbf{1 3})}(\mathbf{n}=\mathbf{3 0 5})$ \\
\hline Fever & $83.0 \%$ & $98.6 \%$ & $43.8 \% \%^{*}, 8.7 \%^{\dagger}$ & $81.1 \%$ \\
\hline Cough & $82.0 \%$ & $59.4 \%$ & $67.8 \%$ & $39.3 \%$ \\
\hline Dyspnoea & $31.0 \%$ & $31.2 \%$ & $18.7 \%$ & Not reported \\
\hline Vomiting & $1.0 \%$ & $3.6 \%$ & $5 \%$ & $15.9 \%$ \\
\hline Diarrhoea & $2.0 \%$ & $10.1 \%$ & $3.8 \%$ & $49.5 \%$ \\
\hline Abdominal pain & Not reported & $2.2 \%$ & Not reported & $6.0 \%$ \\
\hline
\end{tabular}

*On admission. †During course of hospitalisation. COVID-19: coronavirus disease 2019

drug reactions and systemic inflammation in severely ill patients receiving medical treatment. ${ }^{(14)}$ In a report that described autopsy findings, Xu et al noted that while pathological features of ARDS were evident in the lungs, the liver demonstrated only moderate microvascular steatosis with mild lobular and portal activity, which could be either infection-related or due to druginduced liver injury. Flow cytometry analysis of peripheral blood suggested that over-activation of $\mathrm{T}$ cells, manifested by an increase in Th17 and high cytotoxicity of CD8 T cells, contributed to the severe immune injury. ${ }^{(15)}$

\section{IMPACT ON GASTROENTEROLOGY PRACTICE}

It is unlikely that a patient presents solely with Gl symptoms as a manifestation of symptomatic COVID-19 infection. A thorough systemic review should be performed during history-taking, as the cardinal symptoms of fever and respiratory symptoms may be overlooked by the patient. As some Gl patients have chronic or recurrent $\mathrm{Gl}$ symptoms such as irritable bowel syndrome and inflammatory bowel disease, the clinician needs to be mindful of the potential interplay of symptoms, with COVID-19-related symptoms being confused with symptomatic relapse of the underlying $\mathrm{Gl}$ condition, and vice versa. Patients requiring long-term immunosuppression such as those with inflammatory bowel disease and autoimmune hepatitis, as well as patients who are immunocompromised due to chronic disease states such as liver cirrhosis, are a vulnerable group during the COVID-19 pandemic. ${ }^{(3)}$ These patients need to be counselled on risk mitigation and avoidance of exposure. Immunosuppression needs to be tailored on an individualised basis. For infected patients with Gl symptoms, one needs to recognise the possibility that Gl symptoms are part of the disease manifestation and avoid excessive investigations. Noncrucial invasive tests should be deferred till recovery to minimise the risk of disease transmission.

Endoscopy is an intrinsic part of gastroenterology practice. When a country is completely overwhelmed by an outbreak, all elective procedures cease and the limited resources left for GI endoscopy are channelled towards only life-saving emergency therapeutic Gl endoscopy procedures. When the outbreak is still contained and if resources permit, it is possible to continue performing routine $\mathrm{Gl}$ endoscopy procedures that cannot be deferred for too long for uninfected patients. There is a risk of transmission of infection from undiagnosed or pre-symptomatic patients to healthcare professionals during endoscopy. ${ }^{(16)}$ This issue has been addressed by the Chapter of Gastroenterologists,
Academy of Medicine, Singapore, in a recently published guideline. ${ }^{(17)}$ In essence, both upper and lower Gl endoscopy procedures may result in transmission of infection. Upper endoscopy procedures are considered aerosol-generating procedures. Additionally, SARS-CoV-2 can be detected in the faeces of infected patients, raising the concern of a faecal-oral route of transmission. ${ }^{(9)}$ Thus, precautions taken during the performance of colonoscopy should be the same as those for upper endoscopy. It is important to perform patient screening and risk stratification before listing a patient for an elective procedure. The aim is to identify patients who may potentially be infected or are at risk of being infected, and reschedule their elective endoscopy to a later date when it is clearer that they are not infected. For patients with confirmed or suspected COVID-19 infection, only emergency Gl endoscopic procedures that have an immediate impact on management should be performed.

As procedures could conceivably be performed on asymptomatic undiagnosed patients due to disease spread in the community, and an endoscopy list generally consists of a mix of both upper and lower endoscopy cases, it is recommended that enhanced personal protective equipment (PPE) be used for all GI endoscopy procedures during the COVID-19 pandemic. This enhanced PPE consists of an N95 face mask, eye protection with goggles or a face shield, an operating theatre cap or hairnet, a water-resistant gown, and gloves. Hand hygiene must be practised when PPE is worn and removed. For confirmed or suspected cases of COVID-19 infection needing emergency Gl endoscopy procedures, the use of powered air-purifying respirators (if available), in addition to enhanced PPE, provides an additional safety barrier and should be strongly considered. There must be strict adherence to infection prevention and control measures such as endoscope reprocessing and environmental decontamination within the endoscopy room.

\section{REFERENCES}

1. Wu F, Zhao S, Yu B, et al. A new coronavirus associated with human respiratory disease in China. Nature 2020; 579:265-9.

2. World Health Organization. Coronavirus disease (COVID-19) Weekly Epidemiological Update and Weekly Operational Update. Available at: https:// www.who.int/emergencies/diseases/novel-coronavirus-2019/situation-reports. Accessed April 1, 2020.

3. Wong SH, Lui RN, Sung JJ. Covid-19 and the digestive system. J Gastroenterol Hepatol 2020; 35:744-8.

4. Xiao $\mathrm{Y}$, Pan $\mathrm{H}$, She Q, Wang F, Chen M. Prevention of SARS-CoV-2 infection in patients with decompensated cirrhosis. Lancet Gastroenterol Hepatol 2020; 5:528-9.

5. Ang TL. Gastrointestinal endoscopy during COVID-19 pandemic. J Gastroenterol Hepatol 2020; 35:701-2.

6. Yeo C, Kaushal S, Yeo D. Enteric involvement of coronaviruses: is faecal-oral 
transmission of SARS-CoV-2 possible? Lancet Gastroenterol Hepatol 2020; 5:335-7.

7. Li F. Structure, function, and evolution of coronavirus spike proteins. Annu Rev Virol 2016; 3:237-61.

8. Hamming I, Timens W, Bulthuis ML, et al. Tissue distribution of ACE2 protein the functional receptor for SARS coronavirus. A first step in understanding SARS pathogenesis. J Pathol 2004; 203:631-7.

9. Wang $W, X u$ Y, Gao R, et al. Detection of SARS-CoV-2 in different types of clinical specimens. JAMA 2020; 323:1843-4.

10. Chen N, Zhou M, Dong X, et al. Epidemiological and clinical characteristics of 99 cases of 2019 novel coronavirus pneumonia in Wuhan, China: a descriptive study. Lancet 2020; 395:507-13.

11. Wang D, Hu B, Hu C, et al. Clinical characteristics of 138 hospitalized patients with 2019 novel coronavirus-infected pneumonia in Wuhan, China. JAMA 2020; 323: 1061-9.
12. Guan WJ, Ni ZY, Hu Y, et al. Clinical characteristics of coronavirus disease 2019 in China. N Engl J Med 2020; 382:1708-20.

13. Fang D, Ma J, Guan J, et al. Manifestations of digestive system in hospitalized patients with novel coronavirus pneumonia in Wuhan, China: a single-center, descriptive study. Chin J Dig 2020 Feb 23. Chinese. [Epub ahead of print]

14. Liu C, Jiang ZC, Shao CX, et al. [Preliminary study of the relationship between novel coronavirus pneumonia and liver function damage: a multicenter study]. Zhonghua Gan Zang Bing Za Zhi 2020; 28:107-11. Chinese.

15. Xu Z, Shi L, Wang Y, et al. Pathological findings of COVID-19 associated with acute respiratory distress syndrome. Lancet Respir Med 2020; 8:420-2.

16. Rothe C, Schunk M, Sothmann P, et al. Transmission of 2019-nCoV infection from an asymptomatic contact in Germany. N Engl J Med 2020; 382:970-1.

17. Ang TL, Li JW, Vu CK, et al. Chapter of Gastroenterologists professional guidance on risk mitigation for gastrointestinal endoscopy during COVID-19 pandemic in Singapore. Singapore Med J 2020; 61:345-9. 